\title{
REGIONAL VERSUS DETAILED VELOCITY ANALYSIS TO QUANTIFY HYDRATE AND FREE GAS IN MARINE SEDIMENTS: THE SOUTH SHETLAND MARGIN CASE STUDY
}

\author{
Umberta Tinivella*, Maria F. Loreto, Flavio Accaino \\ Istituto Nazionale di Oceanografia e di Geofisica Sperimentale \\ Borgo Grotta Gigante 42C, Trieste, 34010 \\ ITALY
}

\begin{abstract}
The presence of gas hydrate and free gas within marine sediments, deposited along the South Shetland margin, offshore the Antarctic Peninsula, was confirmed by low and high resolution geophysical data, acquired during three research cruises. Seismic data analysis has revealed the presence of a bottom simulating reflector that is very strong and continuous in the eastern part of the margin. This area can be considered as a useful site to study the seismic characteristics of sediments containing gas hydrate, with a particular focus on the estimation of gas hydrate and free gas amounts in the pore space. Pre-stack depth migration and tomographic inversion were performed to produce a regional velocity field of gas-phase bearing sediments and to obtain information about the average thickness of gas hydrate and free gas layers. Using these data and theoretical models, the gas hydrate and free gas concentrations can be estimated. Moreover, the common image gather semblance analysis revealed the presence of detailed features, such as layers with small thickness characterised by low velocity alternating with high velocity layers, below and above the bottom simulating reflector. These layers are associated with free gas trapped within the hydrate stability zone and deeper sediments. Thus, the use of the detailed and the regional velocity field analysis is important to give a more reliable estimate of gas content in the marine sediments.
\end{abstract}

Keywords: pre-stack depth migration, velocity analysis, gas-phase concentrations

\section{INTRODUCTION}

The presence of a BSR, indicating a relevant gas hydrate reservoir offshore the South Shetland margin (west of the Elephant Island), was discovered during the Italian Antarctic cruise 1989-90, onboard R/V OGS-Explora [1].

This seismic dataset was used in the past to extract detailed velocity information of the shallow structures by using conventional tomographic inversion [2] and jointly tomographic inversion and pre-stack depth migration tool ([3] and [4]). Here, we present a method to obtain a regional seismic velocity field and information about hydrate and free gas presence in the marine sediments, by using a method that is an improvement of the standard analysis of the prestack depth migration output (the Common Image Gather, CIG). The velocity field is obtained with a layer stripping approach and tomographic inversion of the reflections observed in the CIGs [5].

\section{SEISMIC DATA}

A strong BSR was identified on multichannel seismic reflection profiles acquired during the Austral summers 1989/1990 [1] and 1996/1997 [3] on the South Shetland Margin. To better describe the area where the BSR is very strong and continuous, another cruise was carried out to

\footnotetext{
* Corresponding author: Phone: +39040 2140219 Fax +39040 327521 E-mail: utinivella@ogs.trieste.it
} 
acquire detailed bathymetric data, sub bottom profile data and seismic data with a short hydrophone streamer during the Austral summer 2003/2004 [6].

The average seismic velocity in the gas hydrate and free gas zones can be determined by using the pre-stack depth migration and tomographic approach iteratively [5]. We applied this method to the first $16.5 \mathrm{~km}$ of the seismic line IT97206, acquired during the second cruise.

\section{REGIONAL VELOCITY ANALYSIS}

Our main target was to determine the seismic velocity field in order to obtain a seismic image in depth and an estimate of gas hydrate and free gas concentration in the pore space of marine sediments. For this purpose, we decided to use depth migration and tomographic algorithm in the pre-stack domain to determine, iteratively and with a layer stripping approach, both the velocity field and the seismic image in depth. Details about the methodology are described in [2].

We picked the sea floor (Hor 1), a shallow horizon (Hor 2) and two other reflectors (Hor 3 corresponding to the base of layer 2- and Hor 4 the base of layer 3), which, locally, correspond to the BSR (the base of the gas hydrate layer) and the BGR (the base of the free gas layer) respectively. Below the Hor 4 (i.e. in the layer 4), we assumed a velocity at the top of the last layer of $2000 \mathrm{~m} / \mathrm{s}$ and a vertical velocity gradient of $12(\mathrm{~m} / \mathrm{s}) / \mathrm{m}$ : these values were chosen to obtain a satisfactory seismic image in depth. The final velocity model and the final pre-stack depth migration are shown in figure 1 . Note that the final velocity model was vertically and horizontally smoothed to improve the migration and to attenuate lateral velocity variations.

The final velocity field shows a first sub-sea-floor layer characterized by velocities within a range of $1600-1800 \mathrm{~m} / \mathrm{s}$ that increases strongly to about $2250 \mathrm{~m} / \mathrm{s}$ at Hor 2, corresponding to typical velocities of gas hydrate-bearing sediments. The velocity in layer 2 is quite uniform except across CIG 5000 and between CIGs 9900 and 12000 (Fig. 1 , top), where the velocity increases to $2500 \mathrm{~m} / \mathrm{s}$. Layer 3 is characterized by strong lateral velocity variation; in particular we found low velocities (about $1400 \mathrm{~m} / \mathrm{s}$ ) and zones where velocities $(2200-2500 \mathrm{~m} / \mathrm{s})$ are typical of the hydrate-bearing layer. Analysis of the pre-stack depth migrated image suggests that some structural features, such as faults and folds, are localized around velocity anomalies in layers 2 and 3 (Fig. 1).

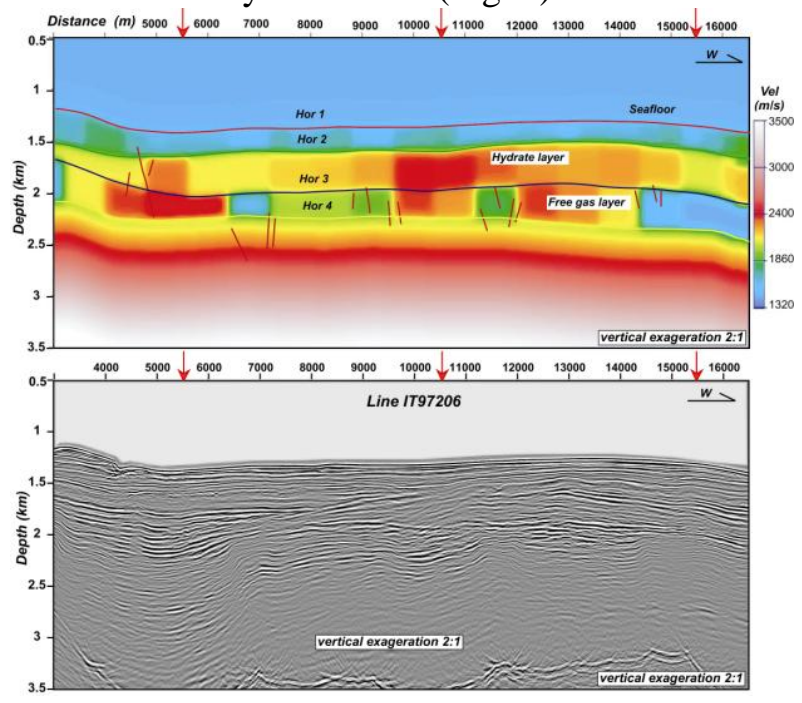

Figure 1: Top: Smoothed velocity field of seismic line IT97206 after tomographic inversion of CIGs.

The distance in meter corresponds to the CIG number. Red arrows correspond to the location of CIGs and CDPs selected for detailed analysis (see text). Bottom: Pre-stack depth migration of seismic line.

\section{DETAILED VELOCITY ANALYSIS}

The pre-stack depth migration (Fig. 1, bottom) indicates that, locally, velocity inversion is absent between layers 2 and 3, even if Hor 3 does correspond to the BSR. To understand the cause of the lack of velocity inversion, we decided to perform detailed velocity analyses at three selected locations (see arrows in Fig. 1) by using both Common Depth Point (CDP) and CIG analyses.

\section{Residual move-out of CDPs}

We show the detailed residual NMO analysis at three selected location along the seismic profile, indicated by red arrows in figure 1 . In figure 2 the NMO corrected CDPs, the interval velocities and the semblances are shown against two-way travel time; the r-parameter represents the time velocity error as obtained by semblance analysis. Note that the semblance energy is quite focused close to the r-parameter zero line, validating the results of the velocity analyses.

All the interval velocity profiles are characterized by alternating high and low velocity layers in both the gas hydrate stability region and below the BSR. 


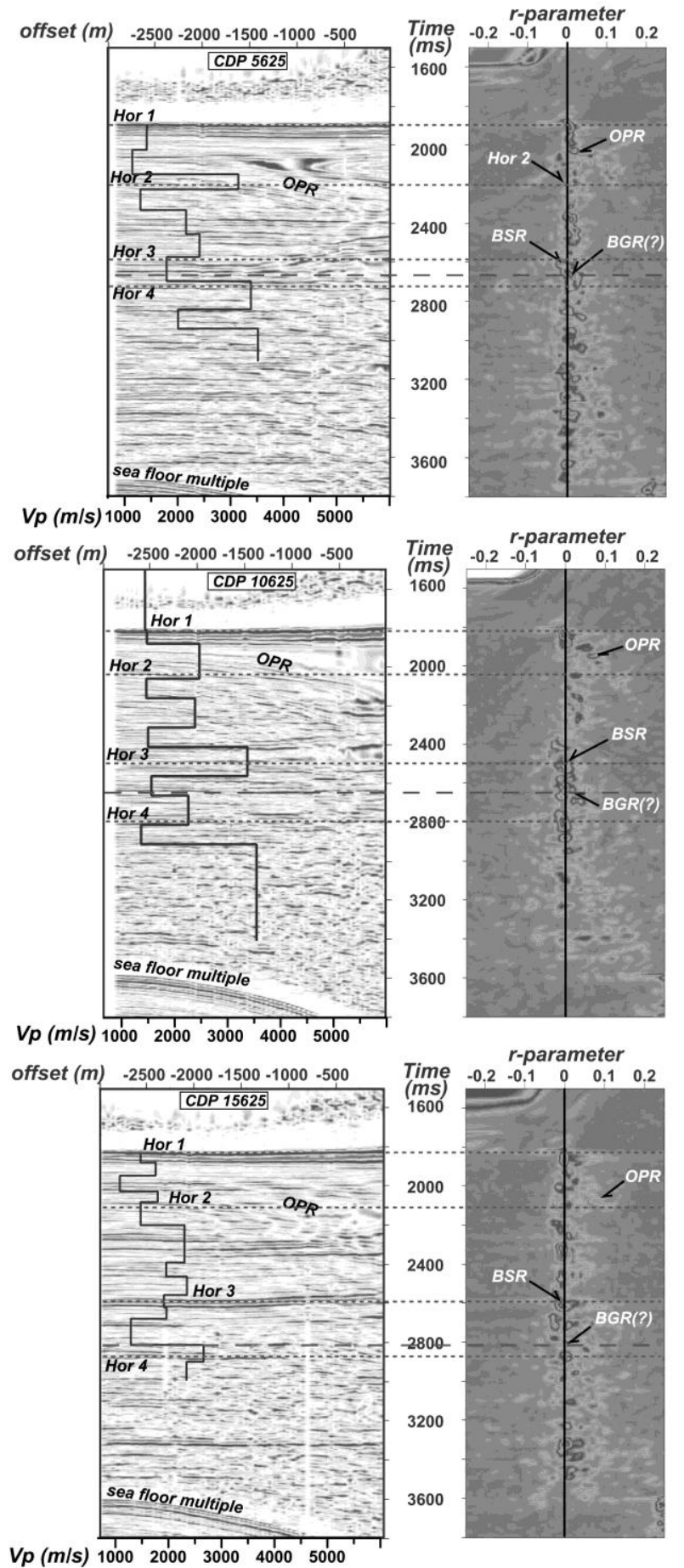

Figure 2: CDPs after residual NMO correction (left) and residual semblance analysis (right) at the three selected locations. The r-parameter represents the time velocity error as obtained by semblance analysis. The profiles are the interval velocities after residual move out analyses. The main horizons are also indicated with dashed gray lines. The BSR and the BGR (horizontal dashed lines) are indicated. OPR: out-of-plane reflection.
Low interval velocities (less than $1500 \mathrm{~m} / \mathrm{s}$ ) are indicated in a few layers with small thickness (about 50-100 m thick). Moreover below the BSR, layers characterised by low velocity are observed at several depths, indicating that the concentration of free gas is variable both along the seismic section and in depth. High velocities are present locally in layer 3 .
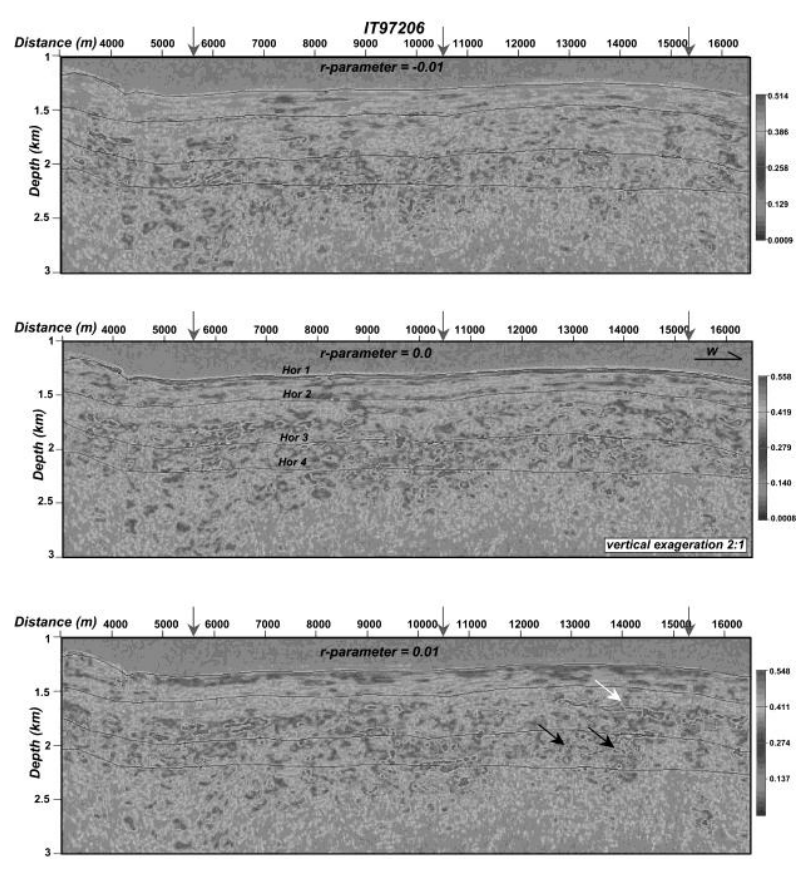

Figure 3: Semblance analysis of CIGs for three values of r-parameter: -0.1 (top); 0.0 (middle); 0.1 (bottom). The distance in meter corresponds to the CIG number. The r-parameter represents the depth velocity error as obtained by semblance analysis. Black lines indicate the four main horizons. White and black arrows are referred to low velocity layers above and below the BSR respectively. The three arrows on the top of each panel indicate the locations of CIGs and CDPs selected for detailed analysis.

\section{Residual move-out of CIGs}

We carried out residual semblance analyses on our final CIGs using the free software Seismic Unix. To visualise the result, three vertical panels are shown in figure 3 , corresponding to three values of the r-parameter: -0.01 (top), 0.0 (middle) and 0.01 (bottom). The images highlight the deviations of the actual seismic velocities from the migration velocity field. In particular, the upper panel furnishes information about the presence of high velocity zones with respect to the migration 
velocity, while the bottom panel indicates the areas where the actual velocity is lower than the migration velocity.

Within layer 2 a prominent reflector (see white arrow in Fig. 3) may be associated with the base of an internal free gas layer. Within layer 3, the residual semblance energy is higher in the positive panel (see black arrows in the lower r-parameter panel). These regions indicate the presence of lowvelocity layers, as also detected by detailed velocity analysis (see Fig. 2). The low-velocity layers characterised by small thickness can be associated with reflections evident in the pre-stack depth migration (see white arrows in Fig. 1). The free gas layer thickness below the BSR probably ranges between about 100-250 $\mathrm{m}$, confirming results of previous studies in the area, in which a variable thickness of the free gas zone was interpreted [4].

Figure 4 shows three selected CIGs at the same location of the CDP analyses (see red arrows in Fig. 1) after the migration performed with constant velocity $(1500 \mathrm{~m} / \mathrm{s}$; left) and the final migration (middle), and the residual semblance analyses (right).

The velocity in the layer 2 is an average between low and high velocities, produced probably by an alternation of free gas and gas hydrate layers. Similar velocity trends above BSRs have been observed by several authors (e.g. [7]).

Comparing the two sets of velocity profiles, the local absence of a velocity inversion between layers 2 and 3 can be understood. In fact, layer 3 is about $300 \mathrm{~m}$ thick, and within it there are several free gas layers characterised by small thickness separated by water saturated sediments, as clearly observed at CIGs 10625 and 15625 (Fig. 4). These low velocity events could correspond to the real BGR (Fig. 4). Finally, the dashed lines in figure 4 show that the positive values of the r-parameter are at the same depth of the low-velocity layers detected by the residual NMO analysis. Thus, even if the r-parameter value can not be directly used to quantify the velocity error, in this case, we can associate these lower velocities to the free-gas bearing sediment layers.
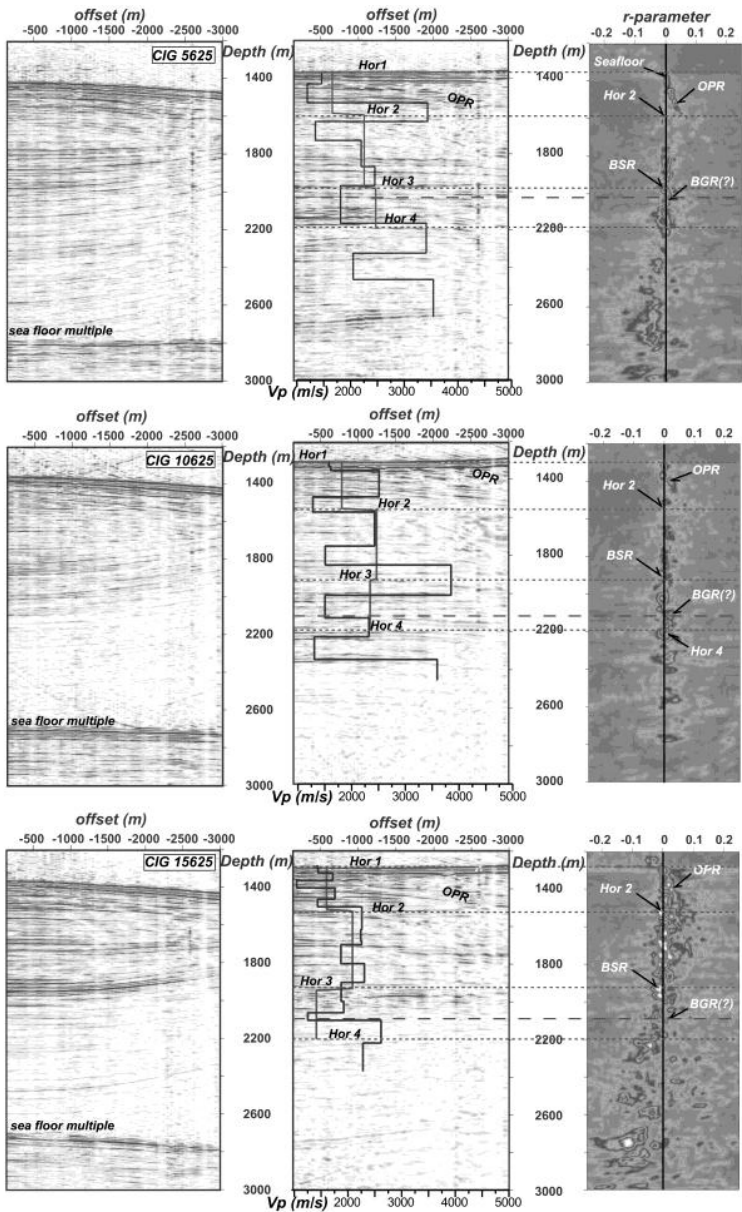

Figure 4: Top: CIG 5625; Middle: CIG 10625; Bottom: CIG 15625. Left panels: CIGs after the pre-stack depth migration performed with a constant velocity field equal to $1500 \mathrm{~m} / \mathrm{s}$. Center panels: CIGs after final pre-stack depth migration. Gray profiles: tomographic velocities. Dark grey profiles: velocity after residual move out analysis. The main horizons are also indicated with dashed gray lines. The BSR and the BGR (horizontal dashed lines) are indicated. OPR: out-of-plane reflection. Right panels: semblance analysis of the selected CIGs, in which the main horizons are indicated.

\section{GAS PHASE CONCENTRATIONS}

The velocity field can be translated in terms of gas hydrate and free gas concentration; for this purpose, we used the methodology already tested in this area (Tinivella, 1999; Tinivella et al., 2002).

The reference curves, i.e. the physical parameters versus depth for water saturated sediments, are the Hamilton curves; we used the average Poisson 
ratio for all sediments equal to 0.435 , obtained by OBS data analysis in the same area [3]. Local analysis suggests that free gas is uniformly distributed in the pore space [3]; so, we evaluated the concentration in this case.

The gas phase section is shown in figure 5. Note that gas hydrate is widely distributed within layer 2 , whereas free gas is present only in localized areas below Hor 3 and is characterised by strong variation of concentration.

Note that the concentration estimations are affected by percentage errors that could be equal to about $\pm 20 \%$ and $\pm 7 \%$ in the case of gas hydrate and free gas, respectively, as established by previous sensitivity tests performed in the same area using the same reference curves [4]. This high value is related to the fact that no drilling data are available and the reference curves are extrapolated using seismic data where the BSR is not present.

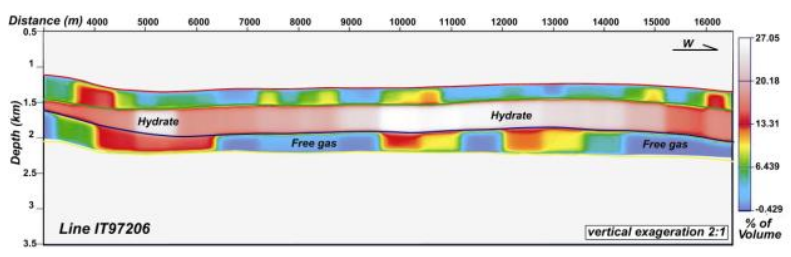

Figure 5: Gas phase concentration obtained from smoothed velocity field considering uniform distribution of both gas phases. The positive and negative values are referred to gas hydrate and free gas concentrations respectively. The four inverted horizons are indicated.

\section{DISCUSSION AND CONCLUSIONS}

The velocity analysis has revealed the presence of three main layers characterising the first kilometer of sediments below the sea floor.

The first layer is characterised by an average velocity of $1735 \mathrm{~m} / \mathrm{s}$ with an average thickness of $250 \mathrm{~m}$, as revealed by both tomographic and semblance analyses. This velocity is higher than the normal compacted accretionary prism sediment velocity. Thus, it could be related to low gas hydrate concentrations in the pore space.

The second layer is characterised by high average velocity $(2220 \mathrm{~m} / \mathrm{s})$ and an average thickness of $345 \mathrm{~m}$. The bottom of this layer in the western part of the section corresponds to the BSR, while in the sedimentary basin the presence of the BSR is not clear because of the seismo-stratigraphy and structural features (faults) affecting sediments; in particular, the faults may act as conduits for gas to escape (Fig. 1). This probably explains why the calculated gas hydrate concentration is lower compared to the western area (Fig. 5). The top of this layer is a very continuous reflector, characterized by normal phase and it simulates the seafloor. On the other hand, the presence of a low velocity strata may be associated to free-gas bearing sediments, or, locally, different lithology.

The layer 3 is characterized by a low average velocity $(1660 \mathrm{~m} / \mathrm{s})$ and an average thickness of $300 \mathrm{~m}$. This low velocity can be interpreted as due to the presence of free gas in the pore space. Moreover, the detailed velocity analyses indicate that the free gas layer is thick about $100 \mathrm{~m}$, resulting thinner than layer 3 (Fig. 3). Finally, figure 4 indicates that free gas is present in one or more layers with small thickness below the BSR between gas free sediments.

In conclusion, the tomographic analysis of CIGs can be considered as a useful tool to determine the velocity field at a regional scale and the seismic image in depth, reducing the human time with respect to other detailed inversion procedures without loss of precision. Moreover, this procedure can be used to provide indications about layers characterized by anomalous physical properties with respect to the surrounding sediments. Thus, by jointly using the tomographic velocity model, the residual semblance analyses of the CIGs and the theoretical models, we can obtain information about the hydrate and free gas thickness and their relative amounts. Finally, velocity models and related gas-phase sections showed that gas is concentrated in different parts of the profile than where hydrate is concentrated. This observation confirm that geological structures and sedimentary processes control the gas and hydrate distribution, as observed along other margins by several authors (e.g. [7]).

\section{REFERENCES}

[1] Lodolo E, Camerlenghi A, Brancolini G. A bottom simulating reflector on the South Shetland margin, Antarctic Peninsula. Antarctic Science 1993;5(2):201-210.

[2] Tinivella U, Lodolo E, Camerlenghi A, Boehm G. Seismic tomography study of a bottom simulating reflector off the South Shetland Islands (Antarctica). In: Henniet J-P, Mienert J editors. Gas hydrate: Relevance to World Margin Stability and Climate Change. Geological Society, London, 1998;137:141-151. 
[3] Tinivella U, Accaino F. Compressional velocity structure and Poisson's ratio in marine sediments with gas hydrate and free gas by inversion of reflected and refracted seismic data (South Shetland Islands, Antarctica). Marine Geology 2000;164:13-27.

[4] Tinivella U, Accaino F, Camerlenghi A. Gas hydrate and free gas distribution from inversion of seismic data on the South Shetland margin (Antarctica). Marine Geophys. Res. 2002;23:109123.

[5] Accaino F, Boehm G, Tinivella U. Tomographic inversion of common image gathers. Firts Break 2005;23:39-44.

[6] Tinivella U., Accaino F, Della Vedova B. Gas hydrates and active mud volcanism on the South Shetland continental margin, Antarctic Peninsula. Geo-Marine Letters 2007;10.1007/s00367-0070093-z

[7] Bunz S, Mienert J, Bryn P, Berg K. Fluid flow impact on slope failure from $3 D$ seismic data: a case study in the Storegga Slide. Basin Research, 2005; 17:109-122. 